\title{
Transport des acides aminés dans l'aire splanchnique par le plasma sanguin et le sang chez le veau préruminant
}

\author{
ML Houlier 1*, P Patureau Mirand 1, D Durand 2, D Bauchart 2, \\ J Lefaivre ${ }^{3}, \mathrm{G}$ Bayle 1 \\ 1 INRA, centre de Clermont-Ferrand, laboratoire d'étude du métabolisme azoté; \\ 2 laboratoire de la croissance et des métabolismes des herbivores; \\ ${ }^{3}$ station de recherches sur la nutrition des herbivores, Theix, 63122 Ceyrat,France
}

(Reçu le 12 septembre 1990; accepté le 25 mars 1991)

\begin{abstract}
Résumé - Trois veaux préruminants porteurs de 3 cathéters permanents (veine porte, veine hépatique, artère mésentérique) et de 2 sondes débitmétriques placées sur la veine porte et sur l'artère hépatique ont reçu des laits de remplacement dont les protéines avaient un transit gastrique lent (régime $L$ ), ou rapide (régime $R$ ). Les teneurs en acides aminés libres du plasma ( $P$ ) et du sang (S), ainsi que les débits sanguins, ont été déterminés avant le repas du matin et pendant les 7 premières heures suivantes. Dans la veine porte, les rapports $\mathrm{P} / \mathrm{S}$ ont été corrélés avec les flux nets plasmatiques au niveau du tractus digestif, pour un grand nombre d'acides aminés. En revanche, dans la veine hépatique, ils ont surtout été corrélés avec les flux nets sanguins hépatiques. Les corrélations entre les quantités libérées par le tractus digestif et celles captées par le foie ont été les plus élevées lorsque le compartiment d'échange considéré a été le sang pour thr et pro, ou le plasma pour cit, ala, val, ile et leu. En conclusion, les échanges d'acides aminés entre le plasma et les globules paraissent relativement faibles et les acides aminés provenant du tractus digestif enrichiraient principalement la fraction plasmatique, alors que les échanges au niveau du foie concerneraient la totalité du compartiment sanguin.
\end{abstract}

veau / acide aminé / sang / plasma / transport

Summary - Amino acid transport through splanchnic bed by plasma or whole blood in preruminant calves. Three preruminant calves were fitted with catheters in portal and hepatic veins and in a mesenteric artery. Two electromagnetic flowmeter probes were clipped around the portal vein and the hepatic artery. The calves were fed either a diet with a low $(L)$ or a high $(R)$ abomasal emptying rate for dietary proteins. Blood flow and free amino acid levels in plasma $(P)$ and blood $(S)$ were determined before the morning meal and during the following $7 \mathrm{~h}$. In the portal vein, for most amino acids $P / S$ ratios were correlated to the net amino acid balance of the digestive tract measured in plasma. By contrast in the hepatic vein, these ratios were mainly correlated to hepatic balance measured in whole blood. Correlations between digestive tract and hepatic balance calculated using either plasma or whole blood pool were different for some amino acids. This suggests that amino acid exchange between plasma and blood cells is low and absorbed amino acids are mainly trans-

\footnotetext{
* Correspondence and reprints
} 
ported to the liver by plasma, whereas whole blood rather than plasma is concerned in amino acid exchanges in the liver.

calt / amino acids / blood / plasma / transport

\section{INTRODUCTION}

Beaucoup de travaux sur les échanges d'acides aminés entre tissus ont été réalisés en supposant que, soit le plasma, soit le sang, était le compartiment permettant ces échanges. De nombreux auteurs considèrent que le compartiment des acides aminés libres plasmatiques est représentatif de celui des acides aminés libres circulants, et que l'étude de ses teneurs peut permettre d'apprécier l'état nutritionnel (Munro, 1972). Toutefois, pour certains, il est préférable de réaliser l'étude sur le sang total (Pion, 1976).

Les résultats des travaux comparatifs antérieurs ont souligné la complexité des phénomènes. Ainsi, selon Elwyn et al (1972), le plasma transporterait les acides aminés des tissus périphériques et du tractus digestif au foie, alors que les globules les véhiculeraient du foie aux tissus périphériques. Par contre, Heitmann et Bergman (1980) ont montré que les échanges de la plupart des acides aminés entre différents organes et le plasma étaient corrélés aux échanges entre ces organes et le sang, mais plus faiblement. Cette sous-estimation était, soit considérable, soit proportionnelle à l'hématocrite, soit limitée selon que les globules contribuaient à ces échanges notablement, de la même façon que le plasma ou beaucoup moins que ce dernier. Afin de préciser le rôle du sang et du plasma dans le transport des acides aminés libres, nous avons étudié, dans des conditions physiologiques chez le veau préruminant, les variations post-prandiales des concentrations en acides aminés libres du sang et du plasma prélevés dans différents vaisseaux irrigant le tractus digestif et le foie après ingestion d'aliments dont les protéines étaient digérées, soit lentement, soit rapidement. Pour tente; d'expliquer les différences observées, nous avons analysé en période post-prandiale les liaisons entre, d'une part le rapport des teneurs plasmatiques en acides aminés libres aux teneurs sanguines, et d'autre part l'intensité des échanges d'acides aminés au niveau du tractus digestif et du foie.

\section{MATÉRIEL ET MÉTHODES}

\section{Animaux et régimes}

Trois veaux préruminants de race Pie Noire pesant entre 40 et $50 \mathrm{~kg}$ ont été logés en case individuelle dans un local ayant une température de $20^{\circ} \mathrm{C}$ et une humidité relative de $80 \%$. Pour mesurer des bilans sanguins d'acides aminés au niveau du tractus digestif et du foie, ils ont été préparés selon le protocole décrit par Bauchart et al (1989). Trois catheters permanents ont été introduits dans la veine porte, dans une veine hépatique et dans une artère mésentérique; 2 sondes d'un débitmètre électromagnétique ont été placées sur la veine porte et sur la branche gauche de l'artère hépatique pour mesurer le débit du sang qui irrigue le tractus digestif et de celui qui arrive au foie. Au niveau de chaque organe, les flux efférent et afférent ont été considérés comme égaux (Durand et al, 1988).

Deux laits de remplacement $L$ et $R$, dont les protéines avaient une vitesse d'évacuation gastrique lente $(L)$ ou rapide $(R)$, ont été distribués aux animaux pendant respectivement 9 et $8 \mathrm{j}$. Ces aliments contenaient $22 \%$ de lipides et $24 \%$ de protéines par rapport à la matière sèche 
(MS). Dans l'aliment $L$, les protéines provenaient en totalité de la poudre de lait écrémé. Dans l'aliment $R$, elles étaient apportées par un concentrat protéique de lactosérum préparé par ultrafiltration et de la poudre de lactosérum (respectivement 96 et $4 \%$ ). Les protéines de l'aliment $R$ étant incoagulables, il en résulte une accélération de leur évacuation gastrique. Les veaux ont été soumis au seau, en 2 repas par jour, apportant au total $58 \pm 9 \mathrm{~g}$ de MS/kg de poids vifo,75. La teneur en MS du lait de remplacement était de $17,5 \%$. Les veaux ont eu un gain de poids vif moyen de $986 \pm 200 \mathrm{~g} / \mathrm{j}$ pendant l'expérience.

\section{Prélèvement et dosages}

Des prélèvements de sang $(7 \mathrm{ml})$ ont été réalisés avant (temps 0) puis $1 / 2,1,2,3,4,5,6$ et $7 \mathrm{~h}$ après le repas du matin. Un millilitre du sang prélevé a été recueilli dans 7 fois son volume d'éthanol $95^{\circ}$ additionné de $2 \%$ de thiodiglycol pour les dosages des acides aminés dans le sang total. L'autre partie a été centrifugée à $1800 \mathrm{~g}$ pendant $10 \mathrm{~min}$ pour obtenir du plasma. Les échantillons ont été conservés à $-15^{\circ} \mathrm{C}$ avant le dosage des acides aminés libras.

Les acides aminés libres du sang ont été extraits à l'éthanol $82^{\circ}$ et l'extrait, purifié par passage sur une résine échangeuse de cations ( $\mathrm{Pa}$ wlak et Pion, 1968). Les acides aminés libres plasmatiques ont été extraits par précipitation à l'acide sulfosalicylique (5\% final) additionné de thiodiglycol ( $2 \%$ final). Les concentrations en acides aminés libres ont été mesurées par colorimétrie à la ninhydrine après chromatographie sur une résine échangeuse de cations à l'aide d'un analyseur automatique (Prugnaud et Pion, 1976). Ces méthodes ne nous ont pas permis de doser quantitativement l'acide glutamique, la glutamine, l'asparagine, le tryptophane et la cystéine dans le sang et le plasma, ni l'acide aspartique et l'arginine dans le sang.

\section{Calculs et analyses statistiques}

Au niveau du tractus digestif, les flux nets sanguins $F_{T D}$ et plasmatiques $f_{T D}$ (mg. $\mathrm{min}^{-1} \cdot \mathrm{kg}^{-1}$ de poids vif) ont été calculés de la manière suivante :

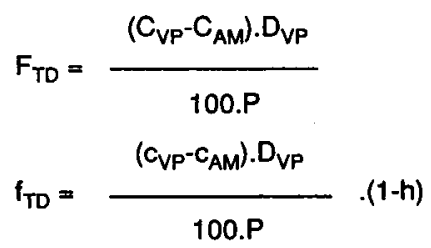

$D_{V P}$ : débit sanguin de la veine porte $(\mathrm{ml} / \mathrm{min})$; $P$ : poids vif de l'animal $(\mathrm{kg}) ; C_{V P}, C_{A M}$, concentrations sanguines dans la veine porte et l'artère mésentérique (mg/100 $\mathrm{ml}$ de sang) respectivement; $c_{V P}, c_{A M}$, concentrations plasmatiques ( $\mathrm{mg} / 100 \mathrm{ml}$ de plasma); $\mathrm{h}=$ hématocrite.

Au niveau du foie, les flux correspondants $\left(F_{F}\right.$ et $\left.f_{F}\right)$ ont été déterminés comme indiqué cidessous :

$$
\begin{aligned}
& F_{F}=\frac{C_{V S} \cdot\left(D_{V P}+D_{A H}\right)-\left(D_{V P} \cdot D_{V P}+C_{A H} \cdot D_{A H}\right)}{100 \cdot P} \\
& f_{F}=\frac{c_{V S} \cdot\left(D_{V P}+D_{A H}\right)-\left(c_{V P} \cdot C_{V P}+C_{A H} \cdot D_{A H}\right)}{100 P} \cdot(1-h)
\end{aligned}
$$

$\mathrm{C}_{\mathrm{vs}}, \mathrm{c}_{\mathrm{vs}}$ : concentrations sanguines et plasmatiques ( $\mathrm{mg} / 100 \mathrm{ml}$ de sang, $\mathrm{mg} / 100 \mathrm{ml}$ de plasma) dans la veine hépatique; $\mathrm{C}_{A H}, \mathrm{C}_{\mathrm{AH}}$ : concentrations sanguines et plasmatiques dans l'artère hépatique respectivement égales à $C_{A M}$ et $C_{A M}$; $\mathrm{D}_{\mathrm{AH}}$ : débit sanguin dans l'artère hépatique, soit deux fois celui mesuré dans la branche gauche $(\mathrm{ml} / \mathrm{min})$.

Afin de comparer les teneurs plasmatiques et sanguines en acides aminés libres, le rapport $P /$ $S$ de la concentration plasmatique $(P)$ de chaque acide aminé à sa concentration sanguine (S) a été calculé, en tous points de la cinétique dans les 3 vaisseaux, avec les 2 régimes. Une analyse de la variance de mesures répétées a été réalisée pour déterminer l'incidence de la nature de l'aliment, du site de prélèvement, de l'animal. L'influence de l'intensité des échanges d'acides aminés au niveau du tractus digestif et du foie sur leurs teneurs plasmatiques et sanguines a été étudiée en recherchant les corrélations entre P/S et les flux nets mesurés dans le sang et le plasma au niveau de chaque organe, ainsi que par la confrontation de leur sens de variation simultané entre 2 prélèvements consécutifs (SAS, 1988). 


\section{RÉSULTATS}

Comparaison des teneurs en acides aminés libres du plasma sanguin et du sang

La somme des teneurs en acides libres du sang ou du plasma a évolué de façon très différente selon la nature du régime (fig 1). Avec l'aliment $L$, les teneurs plasmatiques et sanguines ont peu évolué $(<15 \%)$ : une légère baisse pendant les 2 premières heures a été suivie d'une faible augmentation. L'ingestion de l'aliment $R$ a provoqué un accroissement rapide et important $(>200 \%$ ) des teneurs sanguines et plasmatiques en acides aminés jusque vers

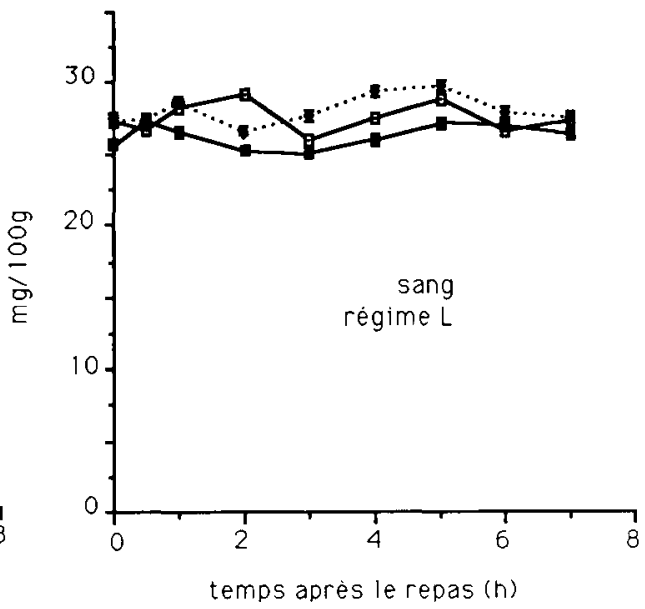

temps après le repas (h)

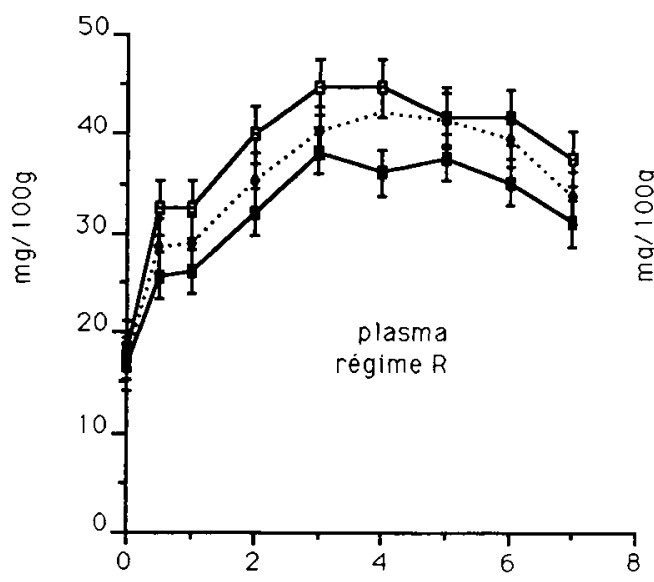

temps après le repas ( $h$ )

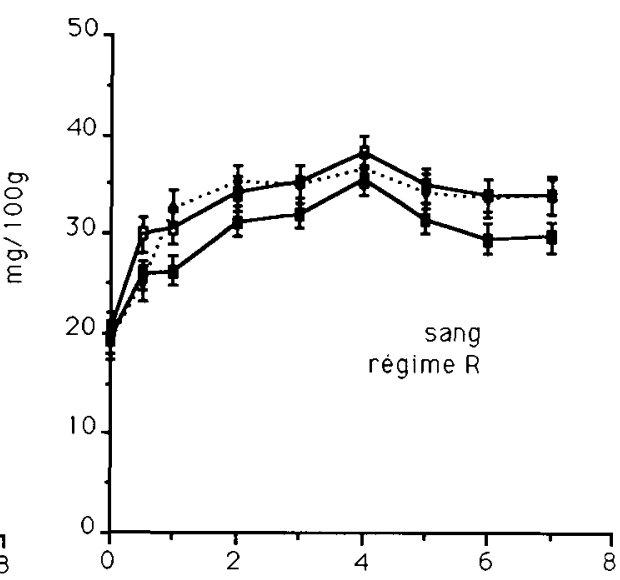

temps après le repas (h)

Fig 1. Cinétiques post-prandiales de la somme des teneurs en acides aminés libres dans la veine

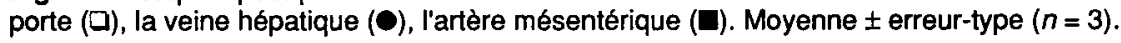


$5 \mathrm{~h}$ après le repas; ensuite ces teneurs ont peu varié dans le sang et ont diminué dans le plasma des 3 vaisseaux. Toutefois, ces variations ont été relativement parallèles dans les 3 vaisseaux, aussi bien dans le sang que dans, le plasma, pour les 2 aliments.

Par contre, les évolutions des concentrations plasmatiques et sanguines, bien que voisines, ont présenté des divergences. Pour la somme des teneurs en acides aminés libres, en acides aminés indispensables et en leucine, le rapport P/S a varié significativement selon la nature du régime, l'animal et le site de prélèvement (tableau I). L'effet nature du régime a aussi été significatif pour la thréonine, la lysine et I'histidine. Les rapports P/S de la somme des teneurs en acides aminés à chaîne ramifiée, de la phénylalanine et de l'alanine dépendaient du site de prélèvement et de l'animal. Pour certains acides aminés : citrulline, glycine, méthionine et tyrosine, des différences entre animaux ont été mises en évidence.

La diversité des valeurs du rapport $\mathrm{P} / \mathrm{S}$ non seulement entre acides aminés mais aussi entre régimes est illustrée dans le tableau II. Ainsi avec le régime $L$, la somme des teneurs en acides aminés a été voisine dans le plasma et le sang des 3 vaisseaux $(0,975<P / S<1,025)$, tout au cours de la cinétique (non montrée). II en est de même pour la somme des teneurs en acides aminés à chaîne ramifiée. En revanche, P/S est resté inférieur à 1 pour la somme des teneurs en acides aminés indispensables, du fait de l'histidine et de la

Tableau I. Effets du régime, du veau et du site de prélèvement sur le rapport des teneurs plasmatiques et sanguines (P/S).

\begin{tabular}{lllll}
\hline Acides aminés & Régime & Veau & Vaisseau & Régime * Vaisseau \\
\hline Thréonine & $* * *$ & $* *$ & NS & NS \\
Sérine & NS & NS & NS & NS \\
Citruline & NS & $* * *$ & NS & NS \\
Glycine & NS & $* * *$ & NS & NS \\
Alanine & NS & $* *$ & $*$ & NS \\
Valine & NS & NS & NS & NS \\
Méthionine & NS & $*$ & NS & NS \\
Isoleucine & NS & NS & NS & NS \\
Leucine & $(*)$ & $* * *$ & $*$ & NS \\
Tyrosine & NS & $* * *$ & NS & NS \\
Phénylalanine & NS & $* *$ & $*$ & NS \\
Ornithine & NS & NS & NS & NS \\
Lysine & $* * * *$ & NS & NS & NS \\
Histidine & $* *$ & NS & $*$ & NS \\
SAA & $* * *$ & $*$ & NS \\
SAAI & $* *$ & $* *$ & $*$ & NS \\
SAACR & NS & $* *$ & $*$ & \\
& & & & \\
\hline
\end{tabular}

NS : non significatif; ( $\left(^{*}\right): P<0,1{ }^{*}: P<0,05 ;{ }^{* *}: P<0,01 ;{ }^{* * *}: P<0,001$; SAA, SAAl, SAACR, somme des teneurs en acides aminés libres totaux, indispensables, à chaîne ramiflée, respectivement. 
thréonine principalement (fig 2a). Avec le régime $R$, ce rapport pour l'ensemble des acides aminés libres a évolué au cours du temps (fig 2b). II a augmenté jusqu'à $3 \mathrm{~h}$ passant de 0,8 à 1,15 en moyenne dans les 3 vaisseaux, s'est maintenu jusqu'à $6 \mathrm{~h}$ puis a diminué, en particulier dans le cas de la thréonine. Quelle qu'ait été la nature du régime, les concentrations sanguines de glycine et d'histidine ont été beaucoup plus importantes que les concentrations plasmatiques. À l'inverse, l'ornithine a eu un rapport nettement supérieur à 1 (tableau II).

\section{Influence de l'intensité des échanges d'acides aminés au niveau du tractus digestif et du foie sur leurs teneurs plasmatiques et sanguines}

Les cinétiques post-prandiales des flux nets d'acides aminés indispensables sont représentées dans la figure 3. Afin de savoir si ces flux peuvent contribuer à expliquer la variabilité des teneurs plasmatiques et sanguines, leur corrélation avec les rapports $\mathrm{P} / \mathrm{S}$ de la veine porte (pour les flux mesurés au niveau du tractus digestif), et de la veine hépatique (pour les flux mesurés au niveau du foie), a été estimée.

Au niveau du tractus digestif, les coefficients de corrélation entre les flux nets plasmatiques et les rapports $P / S$ sont significatifs $(P<0,05)$ pour tous les acides aminés sauf la glycine, l'alanine, la phénylalanine, l'ornithine et la proline. Pour ces acides aminés, les coefficients de corrélation sont significatifs entre le rapport $P / S$ et les flux nets sanguins. Pour la citrulline, la valine, la méthionine et la tyrosine seulement, les corrélations sont significatives à la fois dans le sang et le plasma (tableau III). À l'inverse, au niveau du foie, les coefficients de corrélation sont significatifs entre le rapport $\mathrm{P} / \mathrm{S}$ de la veine hépatique

Tableau II. Rapport P/S pour chaque acide aminé à un temps caractéristique de la cinétique, $3 \mathrm{~h}$ après le repas.

\begin{tabular}{|c|c|c|c|c|c|c|}
\hline \multirow[b]{2}{*}{ Acides aminés } & \multicolumn{3}{|c|}{ Régime $L$} & \multicolumn{3}{|c|}{ Régime $R$} \\
\hline & $\begin{array}{l}\text { Veine } \\
\text { porte }\end{array}$ & $\begin{array}{c}\text { Veine } \\
\text { hepatique }\end{array}$ & $\begin{array}{c}\text { Artère } \\
\text { hépatique }\end{array}$ & $\begin{array}{l}\text { Veine } \\
\text { porte }\end{array}$ & $\begin{array}{c}\text { Veine } \\
\text { hépatique }\end{array}$ & $\begin{array}{c}\text { Artère } \\
\text { hépatique }\end{array}$ \\
\hline Thréonine & 0,835 & 0,838 & 0,764 & 1,170 & 1,150 & 1,194 \\
\hline Sérine & 0,798 & 0,740 & 0,724 & 0,928 & 0,787 & 0,787 \\
\hline Citrulline & 1,160 & 1,138 & 1,134 & 1,379 & 1,287 & 1,364 \\
\hline Glycine & 0,739 & 0,682 & 0,700 & 0,780 & 0,714 & 0,768 \\
\hline Alanine & 1,110 & 1,090 & 1,060 & 1,238 & 1,113 & 1,156 \\
\hline Valine & 1,106 & 1,070 & 1,056 & 1,269 & 1,113 & 1,157 \\
\hline Méthionine & 1,172 & 0,974 & 0,864 & 1,197 & 1,222 & 1,268 \\
\hline Isoleucine & 1,025 & 0,946 & 0,973 & 1,161 & 1,062 & 1,088 \\
\hline Leucine & 0,983 & 0,981 & 0,933 & 1,173 & 1,071 & 1,121 \\
\hline Tyrosine & 1,117 & 1,018 & 0,949 & 1,247 & 1,104 & 1,120 \\
\hline Phénylalanine & 0,918 & 0,977 & 0,964 & 1,246 & 1,060 & 1,075 \\
\hline Ornithine & 1,542 & 1,578 & 1,569 & 1,682 & 1,345 & 1,584 \\
\hline Lysine & 1,077 & 1,207 & 1,085 & 1,455 & 1,300 & 1,346 \\
\hline Histidine & 0,626 & 0,586 & 0,543 & 0,583 & 0,429 & 0,388 \\
\hline SAA & 1,025 & 1,003 & 0,975 & 1,228 & 1,112 & 1,156 \\
\hline SAAI & 0,980 & 0,969 & 0,921 & 1,204 & 1,093 & 1,128 \\
\hline SAACR & 1,042 & 1,010 & 0,995 & 1,198 & 1,082 & 1,123 \\
\hline
\end{tabular}

Moyenne des 3 veaux. SAA, SAAI, SAACR : voir tableau l. 

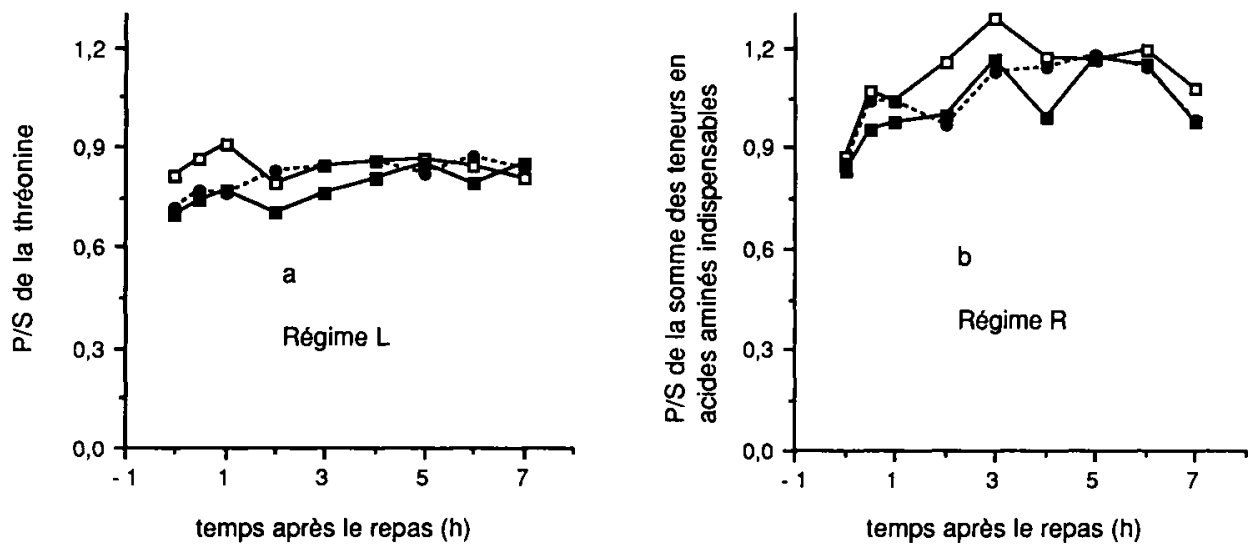

Fig 2. Cinétique du rapport des concentrations plasmatiques aux concentrations sanguines pour la thréonine (a) ou la somme des acides aminés indispensables (b) dans la veine porte ( $\square$ ), dans la veine hépatique (@) et l'artère mésentérique (回). Moyenne \pm erreur type $(n=3)$.

et les flux sanguins pour presque tous les acides aminés (tableau III). En revanche, les corrélations ne sont significatives avec les flux plasmatiques que pour la sérine, l'alanine, l'ornithine, la lysine et la proline.

L'étude des relations entre les sens de variation des flux et ceux du rapport $P / S$ entre 2 prélèvements consécutifs, confirme les liaisons décrites ci-dessus; dans la majorité des cas, il y a un effet significatif du sens des variations des flux sur celui du rapport P/S, c'est-à-dire, dans au moins $70 \%$ des cas, concordance lorsqu'il dépend du flux plasmatique et opposition lorsqu'il dépend du flux sanguin (tableau III). Cela indique aussi que les teneurs plasmatiques en acides aminés indispensables sont plus sensibles à l'afflux alimentaire que celles des autres fractions sanguines. Ce phénomène est confirmé en
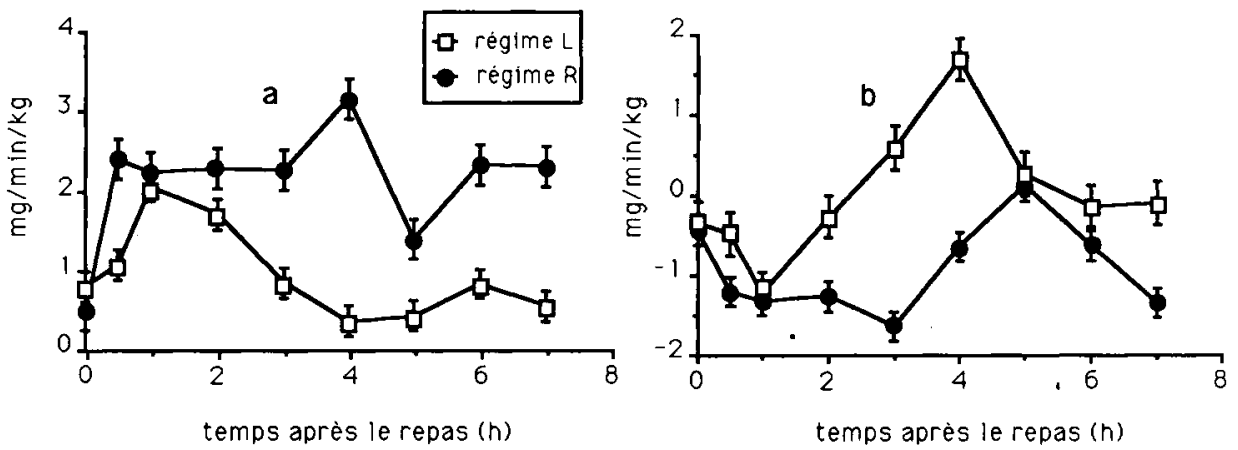

Fig 3. Cinétiques des flux nets plasmatiques de l'ensemble des acides aminés indispensables au niveau du tractus digestif (a) et du foie (b). Moyenne \pm erreur type $(n=3)$. 
Tableau III. Relations entre les rapports $\mathrm{P} / \mathrm{S}$ des concentrations plasmatiques et sanguines des acides aminés libres dans la veine porte ou dans la veine hépatique à leurs flux nets.

\begin{tabular}{|c|c|c|c|c|c|c|c|c|}
\hline & \multicolumn{4}{|c|}{ Veine porte } & \multicolumn{4}{|c|}{ Veine hépatique } \\
\hline & \multicolumn{2}{|c|}{ Plasma } & \multicolumn{2}{|c|}{ Sang } & \multicolumn{2}{|c|}{ Plasma } & \multicolumn{2}{|c|}{ Sang } \\
\hline $\mathrm{n}$ & 54 & 48 & 54 & 48 & 54 & 48 & 54 & 48 \\
\hline Thréonine & ** & 71 & & & & & ** & 64 \\
\hline Sérine & ** & 65 & & & * & & & \\
\hline Citrulline & ** & & $*$ & 75 & & & $* *$ & 75 \\
\hline Glycine & & & $* *$ & 75 & & 71 & $* *$ & 70 \\
\hline Alanine & & & ** & 71 & ** & & & \\
\hline Valine & ** & 75 & ** & 65 & & & ** & 77 \\
\hline Méthionine & $*$ & & ** & & & & ** & 68 \\
\hline Isoleucine & ** & 75 & & & & & ** & 70 \\
\hline Leucine & $* *$ & 71 & & & & & ** & 70 \\
\hline Tyrosine & * & 75 & * & & & & & 70 \\
\hline Phénylalanine & & 69 & ** & & & & & 72 \\
\hline Ornithine & & & ** & & ** & 68 & ** & 68 \\
\hline Lysine & ** & & & & $*$ & & & \\
\hline Histidine & ** & 79 & & & & & ** & 70 \\
\hline Proline & & & ** & 69 & ** & 71 & & \\
\hline SAA & ** & & & & & & & 77 \\
\hline SAAI & ** & 75 & & & & & * & 74 \\
\hline SAACR & $*$ & & & & & & *. & 68 \\
\hline
\end{tabular}

(1) $\mathrm{C}$ : coefficient de corrélation de Kendall ** $P<0,01$; $P<0,05$; (2) $\mathrm{F}$ : fréquence des concordances (niveau plasmatique) ou opposition (niveau sanguin) entre les sens de variations de $P / S$ et ceux des flux entre 2 préleverments consécutifs lorsque l'effet flux sur $P / S$ peut être considéré comme significatif $(P<0,05 ; P<0,01) ;(3) n$ : nombre d'observations; (4) SAA, SAAI, SAACR : voir tableau I.

comparant la composition en acides aminés indispensables des quantités absorbées dans le plasma au niveau du tractus digestif à celle des régimes $L$ et $R$ (fig 4).

\section{Libération des acides aminés par le tractus digestif et leur prélèvement par le foie}

Il existe une corrélation étroite entre les libérations d'acides aminés au niveau du tractus digestif et les prélèvements par le foie; cependant, elle est plus ou moins élevée pour un même acide aminé selon la fraction sanguine observée. Pour la citrulline, l'alanine, la valine, l'isoleucine et la leucine, 40 à $50 \%$ de la variance de leur flux plasmatique au niveau du foie peuvent être attribués à la variation de leur flux plasmatique au niveau du tractus digestif, contre 10 à $25 \%$ de la variance de leur flux sanguin pour ces mêmes acides aminés. À l'inverse, pour la thréonine et la proline, 65 à $80 \%$ de la variance de leur flux sanguin au niveau du foie peuvent être attribués à la variation de leur flux sanguin au niveau du tractus digestif contre 16 à $25 \%$ au niveau plasmatique. Les coefficients de corrélation entre les flux sanguins pour la tyrosine et la phénylalanine sont plus élevés que ceux entre les flux plasmatiques, bien que les écarts soient moins importants que dans le cas précédent. 


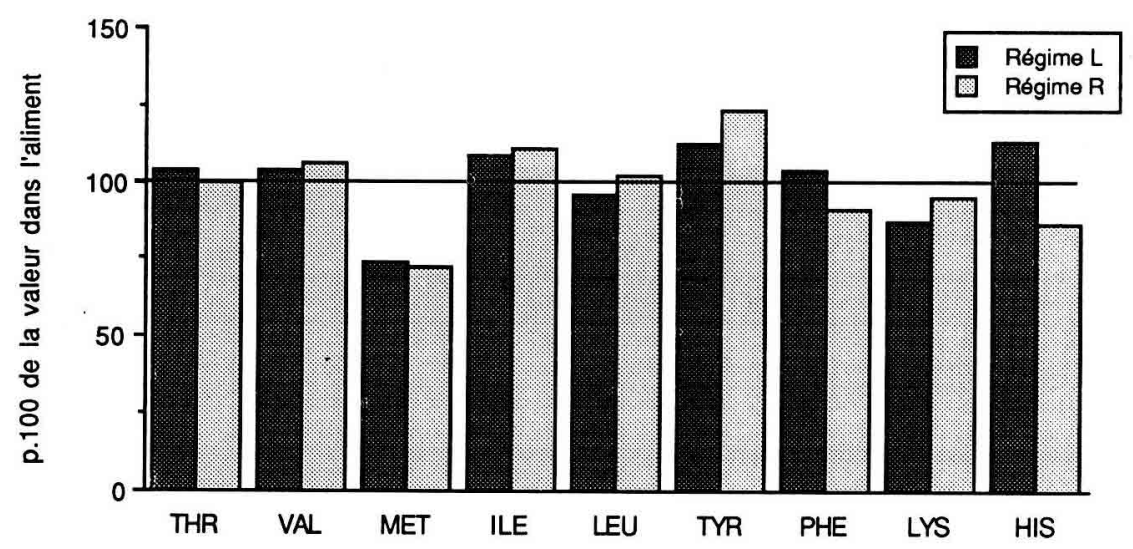

Fig 4. Comparaison de l'équilibre en acides aminés indispensables du mélange absorbé mesuré dans le plasma au niveau du tractus digestif à celui des protéines alimentaires.

\section{DISCUSSION}

Bien que les concentrations plasmatiques et sanguines en acides aminés libres dépendent de nombreux facteurs, un certain nombre d'observations se retrouvent d'une expérience à l'autre. Le veau préruminant nourri avec l'aliment $L$ se comporte comme la plupart des autres espèces. Dans les 3 vaisseaux étudiés, les concentrations de thréonine, sérine, glycine, tyrosine et histidine sont plus élevées dans le sang que dans le plasma. Les teneurs en acides aminés à chaîne ramifiée, alanine et phénylalanine, sont équivalentes dans le sang et dans le plasma. Nos résultats sont tout à fait semblables à ceux d'Elwyn et al (1972), Drewes et al (1977) chez le chien, Keith et al (1977) chez le porc. Heitman et Bergman (1980) chez la brebis, McCormick et Webb (1982) et Koehln (1982) chez le veau. Chez l'homme, Aoki et al (1976) ont observé des résultats similaires, sauf pour la thréonine pour laquelle la différence entre teneurs plasmatiques et te- neurs sanguines n'est pas significative bien que leur rapport soit de 0,87. En ce qui concerne l'ornithine, comme dans les autres travaux chez le veau mentionnés cidessus, les concentrations plasmatiques sont nettement plus élevées que celles du sang, contrairement à ce qui a été décrit chez le chien, le porc, la brebis et l'homme par les auteurs déjà cités. L'ingestion d'un repas d'aliment $R$ qui est rapidement digéré chez le veau préruminant a provoqué, dans les 3 vaisseaux, une augmentation prévisible (Toullec et al, 1983) des concentrations de la plupart des acides aminés dans le sang et surtout dans le plasma. De telles variations ont été rapportées chez le porc, dans le sang porte et carotidien (Rérat et al, 1988). Les concentrations en glycine sanguine sont nettement supérieures aux concentrations plasmatiques. Le rapport P/S de cet acide aminé évolue de la même manière avec les 2 aliments; il n'est pas sensible à l'afflux alimentaire d'acides aminés, ce qui peut en partie s'expliquer par les faibles teneurs en glycine de ces 2 régimes. 
Bien que les rapports des teneurs plasmatiques et sanguines dépendent de l'activité des échanges entre plasma et globules (Hagenfeldt et Arvidsson, 1980), ils ne permettent d'analyser que partiellement les échanges entre plasma, globules, et le rôle des systèmes de transport d'acides aminés de la membrane érythrocytaire. Ainsi le maintien du rapport P/S de la glycine, de la sérine et de l'histidine à un niveau stable et nettement inférieur à 1 dans les 3 vaisseaux, alors que les flux nets varient, pourrait suggérer que les systèmes de transport et de concentration des acides aminés sont particulièrement actifs. Toutefois, dans le cas où initialement la quantité d'acides aminés libres globulaires représenterait $50 \%$ des acides aminés libres sanguins et où le volume plasmatique atteindrait $75 \%$ du volume sanguin, une augmentation de $30 \%$ de la concentration des acides aminés libres plasmatiques, sans modification de celle des globules, n'accroîtrait le rapport P/S que de $13 \%$, ce qui correspond aux fluctuations observées. De plus, il faut noter qu'il s'agit d'acides aminés caractéristiques de systèmes de transport différents (gly, ASC ou asc, $\mathrm{N}$ ou $\mathrm{L}$ ), bien que pouvant partager le même (Collarini et Oxender, 1987; Young et Ellory, 1977; Vadgama et Christensen, 1985). En revanche, l'augmentation des rapports $P / S$ pour les acides aminés à chaîne ramifiée, la phénylalanine, la méthionine, généralement transportés par le système L, ainsi que pour l'alanine, la thréonine, la citrulline et la lysine, tendrait à indiquer que les échanges entre plasma et globules sont peu actifs. En effet, dans le cas où les acides aminés libres des globules ne représenteraient initialement que $20 \%$ des acides aminés libres du sang et où l'hématocrite serait de $25 \%$, une augmentation de $100 \%$ des concentrations plasmatiques, sans modification des concentrations globulaires, n'accroîtrait que de $11 \%$ le rapport $P / S$; or, de telles variations ont été observées. II semblerait donc que chez le veau préruminant, les échanges directs entre globules et plasma soient relativement lents quels que soient les acides aminés. Ce résultat, qui diffère de ceux de Darmaun et al (1989) chez l'homme pour la leucine et dans une moindre mesure pour l'alanine, est néanmoins en accord avec leurs observations pour la glycine.

Cela ne signifie pourtant pas que chez le veau, les globules ne participent pas aux échanges d'acides aminés. En effet, l'analyse des relations entre les variations du rapport P/S et les flux nets d'acides aminés met en évidence leur contribution spécifique dans les échanges hépatiques pour tous les acides aminés, sauf la sérine, l'alanine, la lysine et la proline. En revanche, les échanges du tractus digestif seront plus aisément appréciés par l'étude des teneurs plasmatiques pour l'ensemble des acides aminés indispensables (sauf la phénylalanie), la sérine, la citrulline et les sommes des teneurs en acides aminés libres totaux, indispensables, à chaîne ramifiée (tableau III; fig 4). Ainsi, la contribution du plasma au transport des acides aminés est plus importante dans le sang de la veine porte que dans celui de la veine hépatique; cette observation se vérifie même lorsque les variations relatives des échanges sang-organes sont du même ordre de grandeur aux niveaux digestif et hépatique. Ces résultats sont en accord avec ceux d'Elwyn et al (1972); les acides aminés libres provenant de la digestion de l'aliment sont transportés en grande partie par le plasma sanguin, du tractus digestif au foie, et par le sang, du foie aux tissus périphériques. 


\section{CONCLUSION}

Les variations des teneurs en acides aminés libres présentent des similitudes dans le sang et le plasma, quels que soient les vaisseaux étudiés. Cependant, l'analyse des variations du rapport (P/S) des concentrations plasmatiques aux concentrations sanguines met en évidence des différences qui dépendent du site de prélèvement sanguin, de la nature de l'acide aminé et de l'intensité des échanges entre les organes (tractus digestif ou foie) et le sang ou le plasma. La plupart de ces phénomènes pourraient s'expliquer à partir des 2 constatations suivantes :

- les échanges d'acides aminés entre le plasma et les globules sont peu actifs;

- cependant, les globules joueraient un rôle spécifique au niveau des tissus ou organes; ils participeraient principalement à l'approvisionnement des tissus digestifs en acides aminés; en revanche, ils recueilleraient les acides aminés libérés par le foie.

Cela explique d'une part, pourquoi les variations des teneurs en acides aminés libres plasmatiques de la veine porte sont de meilleurs indicateurs des variations du bilan des tissus digestifs que celles des teneurs sanguines, surtout quand le flux d'acides aminés absorbés est élevé (régime R), et d'autre part, pourquoi les concentrations sanguines de la veine hépatique sont plus étroitement corrélées aux bilans hépatiques d'acides aminés que les teneurs plasmatiques.

\section{RÉFÉRENCES}

Aoki TT, Brennan MF, Müller WA, Soeldner JS, Alpert JS, Saltz SB, Kaufmann RS, Tan MH, Cahill GF (1976) Amino acid levels across normal forearm muscle and splanchnic bed after a protein meal. Am J Clin Nutr 29, 340350
Bauchart D, Durand D, Laplaud PM, Forgez P, Goulinet S, Chapman MJ (1989) Plasma lipoproteins and apoliproteins in the preruminant calf, Bos spp: density distribution, physicochemical properties, and the in vivo evaluation of the contribution of the liver to lipoprotein homeostasis. J Lipid Res 30, 1499-1514

Collarini EJ, Oxender DL (1987) Mechanisms of transport of amino acids across membranes. Ann Rev Nutr 7, 75-90

Darmaun $D$, Froguel $P$, Rongier $M$, Robert JJ (1989) Amino acid exchange between plasma and erythrocytes in vivo in humans. $J$ Applied Physiology 67, 2383-2388

Drewes LR, Conway WP, Gilboe DD (1977) Net amino acid transport between plasma and erythrocytes and perfused dog brain. $A m J$ Physiol 233, E320-E325

Durand D, Bauchart D, Lefaivre J, Donnat JP (1988) Method for continuous measurement of blood metabolite hepatic balance in conscious preruminant calves. J Dairy Sci 71 , 1632-1637

Elwyn DH, Launder WJ, Parikh HC, Wise EM (1972) Roles of plasma and erythrocytes in interorgan transport of amino acids in dogs. Am J Physiol 222, 1333-1342

Hagenfeldt L, Arvidsson A (1980) The distribution of amino acids between plasma and erythrocytes. Clin Chim Acta 100, 133-141

Heitmann RN, Bergman EN (1980) Transport of amino acids in whole blood and plasma of sheep. Am J Physiol 239, E242-E247

Keith MO, Botting HG, Peace RW (1977) Dietary effects on the concentrations of free amino acids in plasma and whole blood of pigs. Can J Anim Sci 57, 295-303

Koehln LL (1982) Movement of plasma free, peptide and serum protein amino acids across the gastrointestinal tract and liver of calves. PhD, Virginia Polytechnic Institute and State University

McCormick ME, Webb KE (1982) Plasma free, erythrocyte free and plasma peptide amino acid exchange of calves in steady state and fasting metabolism. $J$ Nutr 112, 276-282

Munro HN (1972) Free amino acid pools and their role in regulation. In: Mammalian Protein Metabolism (HN Munro, ed) Academic Press, New York, 299-386 
Pawlak M, Pion R (1968) Influence de la supplémentation des protéines de blé par des doses croissantes de lysine sur la teneur en acides aminés libres du sang et du muscle du rat en croissance. Ann Biol Anim Bioch Biophys 8, 517-530

Prugnaud J, Pion R (1976) Dosage des acides aminés dans les aliments. In: Journée de Biochimie : l'analyse des acides aminés. Beckman, Paris $23 p$

Pion R (1976) Dietary effects and amino acids in tissues. In: Protein Metabolism and Nutrition (DJA Cole, KN Bootman, PJ Buttery, D Lewis, RJ Neale, H Swan, eds) Butterworths, Londres, 259-277

Rérat A, Jung J, Kandé J (1988) Absorption kinetics of dietary hydrolysis products in conscious pigs given diets with different amounts of fish protein. 2. Individual amino acids. $\mathrm{Br} J$ Nutr 60, 105-120

SAS (1988) SASISTAT User's Guide. Release 603 edition, SAS Institute Inc, Cary, NC, USA

Toullec $R$, Guilloteau $P$, Patureau Mirand $P$, Sissons JW (1983) Digestion and absorption of protein in the preruminant. In: Protein Metabolism and Nutrition (M Arnal, R Pion, D Bonin, eds), INRA Publications, Paris

Vadgama JV, Christensen HN (1985) Discrimination of $\mathrm{N}_{\mathrm{a}}^{+}$-independent transport systems $L, T$, and ASC in erythrocytes. $J$ Biol Chem 260, 2912-2921

Young JD, Ellory JC (1977) Red cell amino acid transport. In: Membrane Transport in Red Cells (JC Ellory, VL Lew, eds), Academic Press, 301-326 